\title{
BIZILAGUN SAREA: LA SiSTEMATIZACIÓN DE REDES EN el Programa etorkizuna Eraikiz
}

BIZILAGUN SAREA: THE SYSTEMATIC ORGANISATION OF NETWORKS IN THE ETORKIZUNA ERAIKIZ PROGRAMME

\author{
Felix Arrieta*, Bakarne Etxeberria, Ainhoa Izaguirre, Mabel Segú y Martin Zuñiga \\ Departamento de Trabajo Social y Sociología, Universidad de Deusto, Donostia-San Sebastián \\ *autor de contacto: felix.arrieta@deusto.es
}

\begin{abstract}
Resumen: Históricamente, el modelo de servicios sociales de Gipuzkoa se ha caracterizado por un fuerte y estrecho vínculo entre el sector público y el Tercer Sector. Sin embargo, factores tales como el envejecimiento de la población, la crisis económica y la presión a la que el Estado de Bienestar se ve sometido hacen necesaria la reconfiguración del sistema de bienestar. En este sentido, diferentes iniciativas que defienden la importancia del papel de la comunidad en el proceso de desarrollo de políticas sociales han ido adquiriendo mayor protagonismo. Este estudio presenta cinco iniciativas que actualmente se están llevando a cabo en Gipuzkoa con el objetivo de promover nuevas formas de solidaridad vecinal en diferentes ámbitos como pueden ser las personas mayores y las personas en riesgo de exclusión social. Así, el objetivo principal del estudio es precisamente medir el impacto de estas iniciativas en la sociedad gipuzkoana e identificar los factores clave que conducen a su eficacia.
\end{abstract}

Palabras clave: comunidad, iniciativas sin ánimo de lucro, solidaridad vecinal, Estado de Bienestar, política social.

\begin{abstract}
Gipuzkoa's model of Social Services has historically been defined by a strong and close bond between the public sector and the Third Sector. However, the aging of the population, the economic crisis and the pressure to which the Welfare State is been subjected are making inevitable a re-configuration of the welfare system. In this sense, special focus has been paid to different non-profit initiatives that defend the important role of community on the process of social policies development. This study presents five different initiatives that are being carried out in Gipuzkoa in order to promote new forms of neighbourhood solidarity and community volunteering on different spheres such as the elderly and people at risk of exclusion. In this sense, the main aim of the study is to measure their impact on society and to identify key factors that lead to their effectiveness.
\end{abstract}

Key words: community, non-profit initiatives, solidarity networks, welfare system, social politics.

\section{Etorkizuna Eraikiz como elemento de innovación en políticas públicas.}

Etorkizuna Eraikiz es el marco estratégico de políticas públicas puesto en marcha por la Diputación Foral de Gipuzkoa en la legislatura 2015-2019. Este programa, descansa sobre los ejes de la colaboración público - privada y de la experimentación e innovación como elementos tractores del territorio previendo así, desarrollar un proceso por y para la sociedad organizada. Desde esta realidad, la Diputación Foral de Gipuzkoa invitó a entidades, empresas y universidades del territorio a colaborar en los retos estratégicos presentes en el mismo, dibujados de forma compartida.

En este contexto y desde esta filosofía nacen los proyectos que se encuadran bajo el paraguas de 'Bizilagun Sareak' en los que las experiencias piloto llevadas a cabo por entidades públicas y privadas del territorio son acompañadas para su sistematización por la universidad con la idea de extraer los mejores aprendizajes. Más concretamente, esta sistematización permite evaluar de forma rigurosa tanto los factores relacionados con la implementación de los

EUROPEAN
PUBLIC SOCIAL
INNOVATION
REVIEW


programas (factores de éxito, obstáculos, buenas prácticas...) como el impacto de este tipo de actividades en las condiciones de vida de las personas que se benefician de los mismos. De esta forma, las conclusiones de la sistematización permitirán decir si las experiencias llevadas a cabo se pueden extender como forma de implementar políticas a futuro.

El artículo que presentamos a continuación recoge los primeros elementos comunes extraídos de esa sistematización y los lee en forma de innovaciones y aportaciones realizadas al ámbito de la gestión de las políticas públicas.

\section{El reverdecimiento de la comunidad en las políticas públicas}

Los cambios que se están produciendo en las estructuras sociales y económicas de las sociedades Europeas suponen un gran reto para los Estados de Bienestar occidentales, que, cada vez más, se están orientando hacia la búsqueda de nuevas fórmulas de articular los esquemas de bienestar. Esta dificultad reside entre otros, en dos factores fundamentales. Por un lado, la dimensión que están adquiriendo esta serie de cambios, como son el envejecimiento de la población, los cambios en las estructuras familiares y en el mercado de trabajo, los movimientos migratorios $\mathrm{y}$ desplazamientos forzados, etc. Y por otro, las reestructuraciones que los Estados de Bienestar han sufrido, caracterizadas por la mercantilización de derechos sociales, y que han ido minando la capacidad de los Estados de Bienestar. Tal es así, que en la actualidad se ha llegado a plantear que el Estado de Bienestar vive una "Edad de Bronce" (2007-?), precedida por la "Edad de Plata" (19762007) y la de "Oro" (1945-75) en referencia a su progresivo desmantelamiento (Moreno, 2012). La actual tendencia hacia la financiarización de la economía a escala mundial también está impactando negativamente en la capacidad de los Estados de Bienestar para proteger y ofrecer alternativas de vida dignas (Fantova, 2014), así como las políticas de austeridad presupuestaria.

En definitiva, a medida que la complejidad de los retos ha aumentado en términos cuantitativos y cualitativos, la capacidad de los Estados de Bienestar para dar una respuesta efectiva se ha ido reduciendo, hasta el punto de que cada vez más, se habla de la necesidad del aumento de las "colaboraciones de bienestar" (Salamon y Toepler, 2015), para la articulación de sistemas efectivos de bienestar. La crisis parece haber generado una nueva estructura de oportunidad política para la actuación de la sociedad civil organizada (Zubero, 2015:32), y están surgiendo con fuerza los paradigmas de la innovación social (Ghys, 2017), los bienes comunes (Mattei, 2013; Hess y Ostrom, 2016), o el capital social (Putnam, 2000), planteando posibles alternativas desde esferas no siempre institucionales.

Estas colaboraciones de bienestar hacen referencia al reparto de funciones entre diferentes esferas que participan en la provisión de bienestar y cuidado. Acerca de estas esferas, que conforman el denominado como "welfare mix" o agregado de bienestar, existen diferentes aproximaciones y, por tanto, diferentes esquemas. El más conocido quizás sea el propuesto por Esping-Andersen (1993) y denominado "welfare triangle" que está conformado por la familia, el Estado y el mercado. Actualmente, se añade una cuarta esfera, que alude al sector de la iniciativa social o incluso a la comunidad, pero que difiere de un planteamiento a otro. Lo que la actual situación de dificultad pone en relieve es la necesidad de reorganizar y actualizar la función que cumple cada una de las esferas en la provisión de bienestar, incrementando el valor de las menos desarrolladas, sin que supongan una merma en el peso del sector público. Una de las estrategias para la mejora de los sistemas de bienestar reside precisamente en la búsqueda de espacios de acción y colaboración para la esfera de la iniciativa social, la comunidad o el Tercer Sector, e incluso, en determinadas ocasiones, del mercado. A este respecto, no se puede obviar que se puede caracterizar el "welfare mix" como un terreno disputado (Johansson et al. 2015), en el que dependiendo del marco ideológico del que se parta, cada esfera estará llamada a adquirir un determinado papel, que derivará en diferentes modelos de bienestar.

Lo que parece constatable en esta reorganización de los agregados de bienestar a nivel Europeo es un proceso de "nonprofitization" de los Estados de Bienestar, en la medida en que los gobiernos buscan cada vez más la colaboración del Tercer Sector en la provisión de Servicios (Salamon, 2015). Este creciente papel del Tercer Sector en la provisión de bienestar parece ir de la mano de un proceso de "mercantilización" del propio sector (McKay et al. 2015). Pero además de hacia el Tercer Sector, crece también el interés sobre las relaciones y redes comunitarias, que 
plantean quizás una alternativa más compleja desde el punto de vista de la gobernanza.

Ejemplos del creciente interés acerca de la reestructuración del "welfare mix" con un papel más importante de la sociedad civil y las comunidades son proyectos como los que el SIIS ${ }^{1}$ (2017) ha presentado, o estrategias estatales de activación comunitaria que han tenido cierto recorrido en Europa como pueden ser la "Big Society" británica, la "Sociedad Participativa" en Holanda, o el "Secondo Welfare" en Italia. En el plano teórico y discursivo, fueron planteadas como propuestas de descentralización que reivindicaban devolución de poder, funciones o recursos desde el control centralizado hacia instituciones locales o las mismas comunidades (Evans, Marsh, y Stoker, 2013). No obstante, estas estrategias no han estado exentas de polémica y debate, ya que han sido vistas en muchos casos como formas políticamente correctas de introducir recortes y privatizaciones (Fantova, 2014) sin haber logrado un empoderamiento comunitario efectivo.

\section{La comunidad como eje para la innovación}

El de comunidad es un concepto difuso utilizado para definir infinidad de realidades que difieren considerablemente en la realidad que designan. Tanto es así, que los usos que se hacen del término pueden llegar a ser incluso contradictorios (Keller, 2003). Sin entrar en el profundo debate sobre su conceptualización, que resulta ineludiblemente problemático (Subirats y Rendueles, 2016), cabe aclarar que en este trabajo se hace un uso territorializado del concepto. Es decir, se propone un uso "intuitivo" (Rebollo y Morales, 2013) del término que nos remite a una concepción territorial que puede ser asimilable a barrio. Salvando por tanto todas las distancias y siendo conscientes de la simplificación que supone, nos referimos a comunidad como al conjunto de relaciones que se dan en un entorno delimitado que puede ser el barrio o el pueblo en su caso. No es por tanto un barrio una comunidad, sino las relaciones que dentro del barrio se dan, que pueden estar más o menos estructuradas. La comunidad será, por tanto, un sistema de relaciones sociales

${ }^{1}$ El SIIS (centro de estudios y documentación) llevó a cabo un estudio en el que recogió alrededor de 53 experiencias de activación comunitaria y solidaridad vecinal que se han desarrollado en España y Europa.
(Kisnerman, 1990) que se da en un entorno concreto. Cabe aclarar, como indica Fantova (2014) que estas relaciones deben estar motivadas por la lógica de la reciprocidad y no por lógicas del mercado o del derecho.

Desde la perspectiva de este trabajo, la comunidad, es decir, estos sistemas de relaciones localizadas basadas en la lógica del reconocimiento y la reciprocidad, son ejemplos inmejorables de procesos de innovación social. Si seguimos la definición que da Moulaert (2009) sobre la innovación social: "la satisfacción de necesidades humanas alienadas mediante la transformación de las relaciones sociales" (2009: 12), encontramos la justificación para esta categórica afirmación.

En esta misma línea, Edwards-Schachter y Tams (2013), afirman que la comunidad es tanto un medio para la innovación social como el fin en sí mismo de la innovación. En otras palabras, la comunidad es tanto el resultado de esos procesos como un ingrediente para lograrlos. Taylor (2017) señala varios ejemplos en los que la comunidad es la herramienta para la innovación y al igual que hace este último autor, consideramos que los proyectos que se presentan en este trabajo son también ejemplos de innovación social.

$\mathrm{Y}$ se considera así porque la (re)construcción de comunidad puede tener como resultado una red que cubra determinadas necesidades de las personas que participan, como un grupo de consumo, dinámicas para el cuidado de personas mayores, etc. Pero también, independientemente del resultado, porque el proceso en sí mismo de construir comunidad fortalece relaciones, genera nuevas alianzas, y en definitiva, aumenta el capital social a nivel individual entre las personas que participan, y a nivel colectivo, como conjunto de barrio.

Quizás esta concepción de la innovación social no sea la más extendida ya que en la actualidad es notorio que la ola de la innovación social tiene un carácter eminentemente empresarial, y "no es infrecuente que las propuestas de la innovación social tengan un carácter mercantilizador o remercantilizador de la política social" (Fantova, 2014:108). Como señala Mestrum (2016), la innovación social puede ser parte de un proyecto progresista y emancipador, pero la frontera con el pensamiento neoliberal es delgada. 


\section{Proyectos Piloto "Bizilagun Sareak"}

La filosofía de experiencias piloto expuesta previamente, junto con la necesidad de abordar los fenónemos sociales en la nueva época del 'reverdecimiento comunitario', trajo consigo la selección de cinco proyectos experimentales que en la convocatoria de 2017 de Etorkizuna Eraikiz fueron seleccionados bajo el paraguas de 'Bizilagun Sareak' para su puesta en marcha y experimentación durante los próximos dos años.

\section{Lkaleak}

El proyecto Lkaleak parte de la base de que los protagonistas son las personas mayores del barrio junto con otra serie de agentes que puedan tener una función importante en su cuidado y decidan adherirse a la iniciativa (por ejemplo: representantes de asociaciones vecinales, comercios o instituciones públicas). De esta manera, serán los encargados de reflexionar sobre la pertinencia y la posibilidad de (re)construir relaciones y redes de apoyo entre las personas que desarrollan su vida en el barrio y el colectivo de personas mayores en situación de fragilidad para así, ofrecerles atención y apoyo.

Liderado por el Ayuntamiento de DonostiaSan Sebastián y bajo el apoyo de APTES (Asociación para la Promoción de la Tecnología Social) y Emanhar, encargada de la ejecución del trabajo de campo, actualmente se desarrolla a una escala micro en el barrio de Egia (Donostia) aunque nace con la pretensión de servir de base para el desarrollo de dinámicas de similares características en la ciudad y el territorio.

Proyecto Plasma

El proyecto Plasma tiene el objetivo de desarrollar una cultura y una sociedad más solidaria en Oarsoaldea. Para ello, pretende dar visibilidad a las iniciativas de auzolan presentes en el territorio $y$ fomentar estas costumbres, proponiendo iniciativas novedosas a partir de las mismas. Así, todas las personas que forman la iniciativa de auzolan se benefician: tanto el actor que la promueve, como los que la conocen a través de la iniciativa, provocando así interacciones a través de todas las personas que se mueven a su alrededor. Puede decirse, por tanto, que Plasma es un espacio físico, pero también virtual, donde sucede la relación entre los promotores y las personas interesadas que pueden llegar a participar en el mismo.

Desde la coordinación del proyecto se ha puesto en marcha un mapeo de iniciativas comunitarias de auzolan de la comarca, y calendarizado su exposición en distintos lugares. Mediante el conocimiento de estos proyectos de auzolan se promueve la transformación de abajo arriba, así como la interacción entre las personas y la activación del tejido comunitario, básicos en cualquier estructura social.

\section{Autoayuda comunitaria, red vecinal $y$ nuevas fórmulas de voluntariado}

Este proyecto tiene el reto de abrir nuevos caminos para abordar el cuidado de las personas mayores en situación de fragilidad. Su objetivo principal es generar propuestas de autoapoyo comunitario, red vecinal y nuevas fórmulas de voluntariado para el apoyo y el cuidado de las personas mayores en situación de fragilidad en la mancomunidad rural de SAIAZ (Bidani-goiatz, Errezil, Beizama y Albistur) y así, mejorar su calidad de vida abriendo vías para que se dé una mayor participación de estas en el pueblo.

Se plantea un proceso de carácter comunitario y responsabilidad compartida, donde se pretende de manera colectiva trabajar con las personas mayores, las asociaciones y agentes de la mancomunidad, para poder pensar y generar nuevas propuestas, que respondan a las necesidades de las personas mayores en situación de fragilidad en el ámbito rural.

Liderado por la Mancomunidad de Servicios Sociales de Saiaz, cuenta con el asesoramiento técnico de la asociación cooperativa Farapi. Si bien en la actualidad se está aplicando en la mancomunidad de Saiaz (Gipuzkoa) tanto el proyecto como los resultados de este proceso de trabajo son replicables en otras comarcas rurales de Gipuzkoa.

\section{Elkar Ekinez}

El proyecto Elkar Ekinez tiene como objetivo generar experiencias piloto solidarias de encuentro y de generación de vínculos entre diferentes agentes fomentando así, la construcción de nuevas formas de implicación comunitaria desde una perspectiva basada en la deconstrucción de estereotipos, y la construcción de comunidades 
inclusivas teniendo siempre en cuenta la perspectiva de género.

La consecución del objetivo mencionado previamente será posible gracias a una metodología basada en la "Educación Popular". Esta línea de investigación entiende la educación como un proceso que busca formar a las personas en la comprensión de su realidad y de las situaciones y causas estructurales de desigualdad y exclusión ocasionadas por el modelo social actual.

Se propone, por tanto, la realización de tres laboratorios diferentes planteados como espacios generadores de encuentros improbables en donde se den soluciones a necesidades concretas. Están compuestos por dos agentes sociales junto a personas en situación de riesgo y/o exclusión que participen en recursos de carácter colectivo vinculados al sistema vasco de servicios sociales. De tal manera, se plantean los siguientes escenarios:

1. Laboratorio en los barrios del Antiguo y Aiete donde participarán personas usuarias del centro de día de Munto.

2. Laboratorio en Renteria, con personas usuarias del taller ocupacional municipal de Joskintza.

3. Laboratorio en Bergara, con personas en situación de riesgo y/o exclusión atendidas en los servicios sociales de base.

\section{Adinkide/Amigos de los mayores}

El proyecto Adinkide surge en Gipuzkoa en 2017 teniendo su desarrollo como Asociación en diferentes Comunidades Autónomas de España con el nombre de "Amigos de los mayores" y cuyo origen se remonta a Francia en 1946.

El objetivo es procurar apoyo afectivo en el acompañamiento a las personas mayores, preferentemente a aquellas que están en situación de aislamiento y/o tengan dificultades psíquicas o físicas a partir de la generación de redes comunitarias en el tiempo de ocio y respiro.

El proyecto tiene una doble perspectiva de empoderamiento de las redes comunitarias:

1. Por una parte, el proyecto pretende empoderar a las personas mayores que se encuentran solas, desde dos programas atendiendo a criterios de la regularidad en las visitas de los voluntarios.

- Si el acompañamiento requiere regularidad, acompañamiento a domicilio: personas solas en sus domicilios $o$ en residencias que necesitan y por otra establecer redes de voluntarios que puedan ofrecer acompañamiento regularmente.

- Si el acompañamiento es más esporádico y menos regular, Grandes Vecinos, dirigido a personas mayores a modo de prevención para poder mantener, reforzar o crear nuevas redes de apoyo con otras personas mayores que están en las mismas circunstancias en el mismo barrio.

2. Por otra parte, el proyecto pretende empoderar a la red de voluntarios, dándoles oportunidad de generar redes con otras personas, bien sean las personas mayores como otros voluntarios de la organización.

Esta buena práctica es replicable en otros Municipios y otros Países tal y como se ha comprobado.

\section{Metodologia para el seguimiento y sistematización de los proyectos}

El proceso de seguimiento y recogida de información llevado a cabo ha seguido un enfoque cualitativo, centrado en la reflexión sobre la propia práctica. Para ello, además de una labor de acompañamiento individual y enlace entre las diferentes experiencias y el marco "Etorkizuna Eraikiz", se han llevado a cabo dos grupos de discusión, definidos como "espacios de reflexión".

Estos espacios han contado con la participación de los cinco proyectos participantes mediante las personas responsables de los mismos, y las personas encargadas de su seguimiento en la Universidad. La tabla 1 recoge la información principal en lo que a participación y duración respecta. 
Tabla 1. Participantes y duración de los grupos de discusión

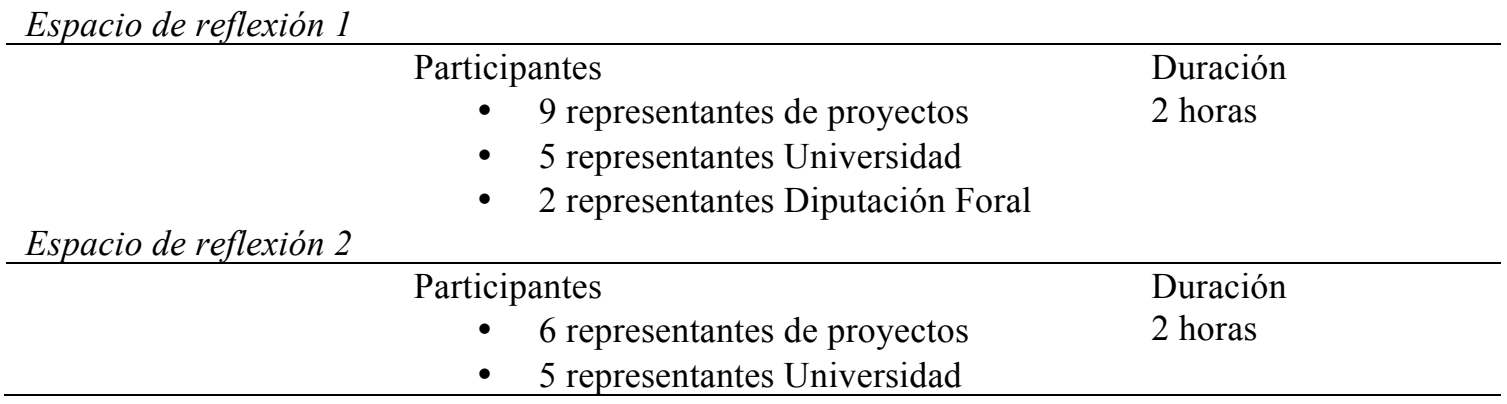

Fuente: Elaboración propia.

Cada uno de los espacios de reflexión, llevados a cabo en momentos bien diferenciados, ha tratado un tema diferente en relación al proceso de los proyectos: mientras que el primero de ellos se ha centrado en la cuestión de cómo se ha entendido e integrado a la comunidad en los proyectos; el segundo, ha hecho especial hincapié en las herramientas y procesos que han seguido para la sistematización del proceso. Ambos temas han sido definidos como centrales en la convocatoria financiadora de los proyectos.

\section{Aprendizajes sobre el desarrollo de los procesos comunitarios}

Los elementos que a continuación se presentan son el resultado del análisis preliminar de los datos obtenidos y representan las reflexiones sobre temas que han sido identificados como característicos y definitorios de los proyectos "Bizilagun".

\section{El carácter interinstitucional e interprofesional}

La participación y colaboración de diferentes instituciones público- privadas a lo largo del proceso es una característica de los proyectos comunitarios y que se ha venido a confirmar en el caso de los proyectos aquí analizados. Esta naturaleza interinstitucional también implica la participación de profesionales de diferentes ámbitos y áreas de conocimiento. Si bien esto ha sido identificado como un elemento enriquecedor, plantea una serie de problemáticas a encarar. La primera de ellas reside en la dificultad de coordinar las instituciones participantes y la búsqueda de sintonía entre ellas. Esto se debe a que la visión y el abordaje del problema que cada una de ellas plantea puede diferir y requerir, por tanto, de un trabajo previo de búsqueda de esa sintonía. A este respecto, se ha destacado el valor de construir un lenguaje común para el desarrollo del proyecto. Esto se traduce en que se deben aclarar una serie de conceptos para el correcto entendimiento entre todas las partes implicadas.

\section{La singularidad de los procesos}

Se ha reconocido que los proyectos comunitarios, de carácter local, resultan en procesos únicos. Pese a que existan ciertos paralelismos entre entornos próximos entre sí, cada contexto tiene unas necesidades y unos recursos que son ciertamente particulares. Esto implica que lo que en un entorno ha sido reconocido como un elemento favorecedor, en otro haya sido identificado como un obstáculo para el desarrollo del proyecto. En este sentido, se ha manifestado que cada experiencia es única y difícilmente extrapolable. No obstante, de cada experiencia se obtienen una serie de aprendizajes que pueden servir como ejemplo y punto de referencia para futuras acciones.

\section{La importancia de la sistematización para posibilitar el aprendizaje y la replicabilidad}

Si bien la búsqueda de elementos que posibiliten la replicabilidad de los proyectos ha sido un objetivo, casi una exigencia, de la convocatoria que ha financiado los proyectos presentados, se ha constatado la dificultad de dicha tarea. No sólo por la singularidad arriba citada, sino por la dificultad de sistematización de un proceso que ha sido 
definido como "artesano", por su carácter informal, repleto de pequeñas acciones y basado en la construcción de relaciones. Muchas de las acciones y conversaciones que se dan a lo largo del proceso, tienen un nivel de espontaneidad que se escapa de lo planificado. No obstante, si bien no en todos los casos, se han utilizado diversas herramientas para la recogida de la información, tales como la grabación de sesiones planificadas, la recogida de actas, o la utilización de cuadernos de campo.

\section{La ambivalencia de la función de la institución pública}

La naturaleza institucional de los proyectos, en este caso representada por órganos municipales, ha tenido diversos resultados. En el entorno urbano, debido a la distancia y desapego entre instituciones y ciudadanía, que haya sido el gobierno municipal quien liderara el proyecto no ha sido visto como una ventaja. Tanto por desconfianza como por reticencias motivadas por cuestiones ideológicas, la lógica "Top Down" de estos proyectos no ha cuajado en determinados segmentos. Por el contrario, en el ámbito rural, en el que las personas se conocen entre sí y no existe tal distancia frente a la institución, ha sido visto como positivo $\mathrm{y}$ fortalecedor.

\section{La importancia de un espacio de referencia}

Una de las conclusiones compartidas por todos los proyectos ha sido la importancia de que exista un espacio físico de referencia para el desarrollo de proyectos comunitarios. Un punto de referencia que sea estable o permanente y que posibilite el encuentro y la creación del tejido que sostiene las iniciativas comunitarias. Por otra parte, este mismo valor de los espacios ha sido reconocido, no sólo para el desarrollo de los procesos, sino para el desarrollo mismo de relaciones comunitarias. En este sentido, se ha hecho referencia a plazas, portales, bares, comercios, etc. en los que las personas socializan y son claves para el desarrollo de dinámicas comunitarias. Son en definitiva lugares, en cada contexto diferentes, en los que se posibilita que se den esas relaciones que construyen comunidad.

\section{Creencias y valores que dificultan la implicación}

Se ha manifestado que existe cierto miedo al compromiso o a la implicación en los proyectos por parte de la ciudadanía, entre otras razones, por verse envueltos en una dinámica que absorba más tiempo del que puedan dedicar. El miedo a verse "absorbido" por la participación en el proyecto lleva a menudo a adoptar posiciones tibias, y a no involucrarse. Las características de la sociedad actual plantean dificultades para la construcción de comunidad. La falta de tiempo y la pérdida de la conciencia colectiva afectan negativamente a la participación comunitaria. En este sentido, se ha propuesto acotar y clarificar en qué consiste la participación, esto es, marcar límites en la acción, de manera que se facilite la implicación.

\section{La dificultad de asegurar la continuidad del proyecto comunitario}

A menudo, estos proyectos de corte comunitario tienen como objetivo activar redes y relaciones que puedan funcionar de manera autónoma $y$ autosuficiente. Las experiencias aquí presentadas muestran sus dudas respecto a esta posibilidad ya que si los proyectos no cuentan con recursos destinados exclusivamente a ello, la continuidad no se ve asegurada. Sin duda alguna, ésta sostenibilidad de los proyectos más allá de lo que dura la vinculación formal o contractual de las entidades con un entorno determinado es una cuestión clave sobre la que reflexionar.

\section{Discusión}

Tal y como se ha mencionado al inicio del presente artículo, es visible el reverdecimiento que la comunidad y las redes vecinales están tomando en el ámbito de las políticas públicas. Esta llamada a la comunidad, que en ocasiones puede ser más simbólica (Del Fresno y Segado, 2013) que efectiva, hace que los proyectos que se presentan sean testigo de la creencia y la voluntad que existe en el territorio de Gipuzkoa acerca de la potencialidad de la comunidad como recurso para dar respuesta a determinadas necesidades sociales. En este sentido, los proyectos que se analizan responden a este interés que parte tanto de la esfera política, como de la propia iniciativa social y comunitaria. Autores de referencia en el estudio de las sociedades occidentales (Bauman, 2003; 
Sennet, 2000) también han dado cuenta de como, en contra de la lógica del capitalismo, está aflorando un sentimiento de comunidad o de necesidad de comunidad. Pese a este doble reverdecimiento, político y social, cabe preguntarse si verdaderamente la comunidad es una alternativa, y si lo es, hasta dónde puede llegar, o cuáles son sus límites.

A la luz de lo experienciado en los proyectos "Bizilagun Sareak", es inevitable el debate en torno al papel de la comunidad en el siglo XXI. La enorme complejidad y diversidad que caracterizan a las sociedades en la actualidad hacen de este tipo de propuestas un camino nada fácil. No obstante, esto también ofrece una nueva ventana de posibilidad, que no es otra que la de construir comunidad en base a nuevos principios, más integradores y más humanistas. A lo largo del desarrollo de los proyectos aquí presentados, de la misma forma que se han identificado unos aprendizajes claros, se han visibilizado $y$ experienciado también ciertas dificultades que representan verdaderos retos. Estos retos, están íntimamente ligados con la capacidad real o funcional que la comunidad puede tener en la sociedad occidental del siglo XXI. Nos referimos a la sostenibilidad de los proyectos, a la implicación efectiva de los vecinos o habitantes de un entorno concreto, y a la posibilidad de replicar o escalar proyectos de estas características.

El reto de la sostenibilidad emerge de la dificultad que supone que, una vez finalizada la vinculación formal o contractual que ha unido a las entidades con un entorno determinado para el desarrollo del proyecto, su funcionamiento autónomo sea efectivo. Las experiencias aquí presentadas muestran sus dudas respecto a esta posibilidad y se plantean las siguientes cuestiones: ¿es posible que las iniciativas subsistan sin el apoyo de instituciones públicas o entidades privadas? ¿es necesaria cierta vigilancia o seguimiento?

Cuando decimos implicación efectiva nos referimos precisamente a que adoptar el compromiso de participar en un proyecto de forma voluntaria no siempre es sencillo, y menos aún, si tal y como se ha señalado en los proyectos, se carece de tiempo e incluso valores que lo posibiliten. Se ha manifestado que existe cierto miedo al compromiso o a la implicación en los proyectos por parte de la ciudadanía, entre otras razones, por verse envueltos en una dinámica que absorba más tiempo del que puedan dedicar. Ante esta realidad, se plantea la pregunta de ¿cómo conseguir la implicación y participación de la comunidad? Y, por tanto, ¿que fórmulas de participación pueden resultar atractivas?

Finalmente, respecto a la replicabilidad, los proyectos expuestos se han caracterizado por su carácter local, muy influenciados por las particularidades y singularidades del entorno. Lo que en algunos casos ha sido determinante, en otros ha resultado ser irrelevante. Esto da muestra de la importancia de realizar un trabajo muy "artesano", centrado en las capacidades y necesidades de cada contexto. Ante esta reflexión, se plantea la duda de si verdaderamente es posible replicar un proyecto de estas características. Sin resolver de manera completa dicho dilema, los elementos descritos previamente hacen deseable y posible que proyectos de estas características se reproduzcan de manera similar en distintos lugares de nuestro territorio.

\section{Referencias}

Bauman, Z. (2003). Comunidad. Madrid: Siglo XXI de España Editores, S.A.

Edwards-Schachter, M., y Tams, S. (2013). How Empowering is Social Innovation? Identifying Barriers to Participation in Community-driven Innovation. In: Mulgan, J., ed. 'Social Frontiers: the next edge for social innovation research' conference, 2013-11-14 - 2013-11-15. London, U. K.: Nesta.

Esping-Andersen, G. (1993). Los tres mundos del Estado del bienestar. Valencia: Alfons el Magnanim.

Evans, M., Marsh, D., y Stoker, G. (2013). Understanding localism. Policy Studies, 34(4), 401-407.

Fantova, F. (2014). Diseño de políticas sociales. Madrid: CCS.

del Fresno, M., y López, A. (2013). Trabajo social con comunidades en el siglo XXI. Madrid: Universitas.

Ghys, T. (2017). Analysing social innovation through the lens of poverty reduction: five key factors. European Public \& Social Innovation Review, 2(2), 1-14.

Hess, C., y Ostrom, E. (2016). Introducción: una visión general de los bienes comunes del conocimiento. En C. Hess, y E. Ostrom, Los bienes comunes del conocimiento (págs. 27-50). Quito: IAEN. 
Itulua-Abumere, F. (2013). The Impact of Community Care Policy on Older People in Britain. 1970s-1990s. Open Journal of Social Science Research, 1(4), 94-98.

Johansson, H., Arvidson, M., y Johansson, S. (2015). Welfare mix as a contested terrain: Political positions on government-non profit relations at national and local levels in a social democratic Welfare State. Voluntas, 26, 1601-1619. Doi: 10.1007/s11266-015-9580-4

Keller, S. (2003). Community: pursuing the dream, living the reality. Princeton, N.J., Princeton University Press.

Kisnerman, N. (1990). Comunidad (Teoría y Práctica del Trabajo Social). Buenos Aires: Humanitas.

Mattei, U. (2013). Bienes comunes. Bolonia:Trotta.

McKay, S., Moro, D., Teasdale, S., y Clifford, D. (2015). The marketisation of charities in England and Wales. Voluntas, 26, 336-354. Doi: 10. 1007/s11266-013-9417-y

Mestrum, F. (2016). Los comunes sociales. Ideas para la justicia social en el pos-neoliberalismo. Petaling Jaya: Gerakbudaya Digital Sdn Bhd.

Moreno, L. (2012). La Europa asocial. Barcelona: Ediciones Península.

Moulaert, F. (2009). Social innovation: institutionally embedded, Territorially (re)produced. En D. MacCallum, F. Moulaert, J. Hillier, \& S. Haddoc, Social innovation and territorial development (págs. 11-24). Surrey: Ashgate.

Putnam, R. (2003). El declive del capital social. Barcelona: Galaxia Gutemberg.

Rebollo, O., y Morales, E. (2013). Hacia una política pública de acción comunitaria: límites y oportunidades. En R. Canal, Ciudades y pueblos que puedan durar. Políticas locales para una nueva época (págs. 303-316). Barcelona: Icaria.

Salamon, L.M. (2015). Introduction: The nonprofitization of the welfare state. Voluntas, 26, 2147-2154. Doi: $10.1007 / \mathrm{s} 11266-015-9638-3$

Salamon, L.M., y Toepler, S. (2015). Government-nonprofit cooperation: Anomaly or necessity? Voluntas, 26, 2155-2177. Doi: 10.1007/s11266-015-9651.

Sennett, R. (2000). La corrosión del carácter. Barcelona: Editorial Anagrama.

Subirats, J., y Rendueles, C. (2016). Los (bienes) comunes ¿oportunidad o espejismo?. Barcelona: Icaria

SIIS Centro de Documentación y Estudios. (2017). Activación comunitaria y solidaridad vecinal. Tendencias y buenas prácticas $=$ Aktibazio komunitarioa eta auzo-elkartasuna. Joerak eta jardunbide egokiak. DonostiaSan Sebastián: Diputación Foral de Gipuzkoa.

Taylor, G. (2017). Social innovation and participatory action research: A way to research community? European Public \& Social Innovation Review, 2(1), 17-33.

Zubero, I. (2012). De los $<<$ comunales $>>$ a los $<<$ commons $>>$ : la peripecia teórica de una práctica ancestral cargada de futuro. Documentación social, 165, 15-48. 